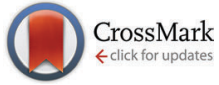

Cite this: Phys. Chem. Chem. Phys., 2015, 17, 6896

Received 30th December 2014 Accepted 29th January 2015

DOI: $10.1039 / c 4 c p 06105 e$

www.rsc.org/pccp

\section{The dual role of palladium in enhancing the photocatalytic activity of CdS dispersed on NaY-zeolite $\dagger$}

\author{
R. Sasikala, ${ }^{\star a}$ A. P. Gaikwad, ${ }^{a}$ V. Sudarsan, R. Rao, ${ }^{b}$ Jagannath, $^{c}$ B. Viswanadh ${ }^{d}$ and \\ S. R. Bharadwaj ${ }^{a}$
}

\begin{abstract}
A stable photocatalyst, CdS dispersed on zeolite with $\mathrm{Pd}$ as both the dopant and the co-catalyst, has been developed. Enhancement of photocatalytic activity for hydrogen generation is observed for CdS when doped with palladium and dispersed on NaY-zeolite (CdPdS-Z). A further increase in the photocatalytic activity of CdPdS-Z is observed when palladium is added as a co-catalyst (Pd-CdPdS-Z). $\mathrm{Cd}_{0.95} \mathrm{Pd}_{0.05} \mathrm{~S}-\mathrm{Z}$ is synthesized via a facile soft chemical route and the $\mathrm{Pd}$ co-catalyst is loaded onto the composite using a wet impregnation method. This composite catalyst exists as two phases consisting of CdPdS and zeolite and CdPdS exists as a highly dispersed phase on zeolite as revealed by TEM studies. The $\mathrm{Pd}$ doped $\mathrm{CdS}$-zeolite composite exhibits increased visible light absorption indicating the alteration of the band structure of CdS as a result of doping. Time resolved fluorescence studies reveal that the lifetime of the charge carriers is higher in the composites than in pure CdS. A detailed characterization using XRD, Raman and X-ray photoelectron spectroscopy indicates that Pd has substituted for Cd in the CdS lattice and Pd exists in the $\mathrm{Pd}^{2+}$ oxidation state. Solid state MAS NMR studies indicate that an interaction exists between $\mathrm{CdS}$ (or CdPdS) and zeolite at the interface and Cd selectively interacts with Al of the zeolite framework. The photocatalytic activity of the Pd-CdPdS-Z catalyst remains unchanged with repeated cycles. Characterization of the used catalyst indicates that it is stable under the present experimental conditions. The enhanced photocatalytic activity of $\mathrm{Pd}-\mathrm{CdPdS}-\mathrm{Z}$ is attributed to the enhanced visible light absorption arising due to Pd doping and increased lifetime of the photogenerated charge carriers assisted by zeolite and the Pd co-catalyst. This study highlights the multiple roles played by palladium in enhancing the photocatalytic activity of the CdS-zeolite composite.
\end{abstract}

\section{Introduction}

Cadmium sulfide based photocatalysts are studied extensively for photocatalytic hydrogen generation from water using visible light. Though CdS is a visible light active photocatalyst, pure CdS has poor photocatalytic activity for hydrogen production in

\footnotetext{
${ }^{a}$ Chemistry Division, Bhabha Atomic Research Centre, Trombay, Mumbai 400085 India. E-mail: sasikala@barc.gov.in; Fax: +91 22 25505151, +91 22 25519613; Tel: +912225592279

${ }^{b}$ Solid State Physics Division, Bhabha Atomic Research Centre, Trombay, Mumbai 400 085, India

${ }^{c}$ Technical Physics Division, Bhabha Atomic Research Centre, Trombay, Mumbai 400 085, India

${ }^{d}$ Material Science Division, Bhabha Atomic Research Centre, Trombay, Mumbai 400 085, India

$\dagger$ Electronic supplementary information (ESI) available: S1. Details of the photocatalytic reactor and irradiation chamber. Fig. S2. Emission profile of the fluorescent lamp used for photocatalysis. Fig. S3. More TEM images and SAED pattern of 20CdPdS-Z. Fig. S4. XRD patterns of fresh and used catalysts. See DOI: 10.1039/c4cp06105e
}

the absence of sacrificial reagents. One of the reasons for the poor activity is that the lifetime of the photogenerated charge carriers is too small to reach the surface of the photocatalyst and initiate the reduction of water. Besides, in the absence of suitable sacrificial reagents, photocorrosion of CdS occurs, making it unstable. Often, sacrificial reagents such as sodium sulfide and sodium sulfite are used to increase the stability and lifetime of the charge carriers for CdS based systems. ${ }^{1}$

The lifetime of the photogenerated charge carriers can be improved by preparing a composite of the photocatalyst with other semiconductors having suitable conduction band (CB) and valence band (VB) potentials. In such composites, the transfer of charges from one semiconductor to the other can take place, resulting in an increased lifetime of the charge carriers. Photocatalytic activities of composites of $\mathrm{CdS}$ with $\mathrm{TiO}_{2}{ }^{2} \mathrm{ZnS},{ }^{3}$ polyaniline, ${ }^{4} \mathrm{ZrO}_{2},{ }^{5}$ zeolite, ${ }^{6} \mathrm{ZnO},{ }^{7} \mathrm{CdO},{ }^{8,9}$ graphene ${ }^{10-15}$ etc. have been reported and in most cases an increased activity is observed for the composites compared to single phase CdS. The reason attributed for the enhancement is the improved surface area and 
better separation of photogenerated charge carriers. Similarly, it is reported that the $\mathrm{N}$ doped graphene-CdS composite shows enhanced photocatalytic activity compared to pure CdS. ${ }^{16}$ In this case, $\mathrm{N}$ doped graphene acts as a co-catalyst, enhances charge separation and improves photocatalytic activity. A solid solution of $\mathrm{Zn}_{0.8} \mathrm{Cd}_{0.2} \mathrm{~S}$ with Pt as the co-catalyst is found to be an efficient photocatalyst for hydrogen generation from water. ${ }^{17}$ The enhanced activity is attributed to a relatively negative conduction band and increased visible light absorption by the solid solution. Composites of $\mathrm{ZnO}$ with $\mathrm{CdS}$ and carbon materials like graphene, $\mathrm{C}_{60}$, carbon nanotubes, etc. have been reported to enhance the photocatalytic activity for dye degradation and minimize photocorrosion. ${ }^{18}$ The reasons for the enhanced photocatalytic activity are the increased lifetime of the charge carriers and improved visible light absorption.

In the present work, CdS is doped with Pd with the aim of increasing the visible light absorption. Further, it is dispersed on zeolite and Pd is impregnated on the surface as a co-catalyst to improve the separation of charges. The $\mathrm{Pd}^{2+}$ dopant can modify the bandgap whereas the surface $\mathrm{Pd}^{0}$ (impregnated as the co-catalyst) can separate the charges efficiently. Though previous reports are available on the role of surface Pd as a co-catalyst in enhancing the charge separation, the role of $\mathrm{Pd}$ as a dopant in CdS-zeolite composite has not been investigated. The present study highlights the dual role of Pd in enhancing the photocatalytic activity of CdS.

\section{Experimental}

\subsection{Synthesis of CdS-zeolite and Pd doped CdS- zeolite composites}

CdS (10, 20, 30 and $50 \%$ by weight) was loaded onto Na-Y-zeolite (named $x \mathrm{CdS}-\mathrm{Z}$, where $x=10,20,30$ and 50) using an impregnation method. Commercially available zeolite (Süd-Chemie India Ltd) was used after drying in an oven at $120{ }^{\circ} \mathrm{C}$ for $6 \mathrm{~h}$. Aqueous cadmium chloride solution (water + ethanol, 1:1 ratio) was stirred with zeolite powder. This suspension was treated with a calculated amount of $\mathrm{Na}_{2} \mathrm{~S}$ dissolved in a water-ethanol mixture (1:1 ratio) under constant stirring for $1 \mathrm{~h}$. The precipitate was centrifuged, washed with water and ethanol three times each followed by heating the dried powder at $350{ }^{\circ} \mathrm{C}$ for $4 \mathrm{~h}$. For the synthesis of palladium doped ( $5 \%$ by atomic weight) $\mathrm{CdS}$ (20CdPdS-Z), a similar procedure was adopted except that a mixed aqueous solution of $\mathrm{CdCl}_{2}$ and palladium chloride was used for the synthesis. Pure CdS was prepared by the reaction of cadmium chloride with sodium sulfide under similar conditions described above. Pd metal (0.5\% by weight) was loaded onto CdPdS (Pd-CdPdS), and 20CdPdS-Z (Pd-20CdPdS-Z) using a wet impregnation method. Sample powders were kept in contact with aqueous palladium chloride solutions and evaporated to dryness under constant stirring followed by heating in air at $350{ }^{\circ} \mathrm{C}$ for $2 \mathrm{~h}$. No reductive treatment was given to these catalysts before the photocatalysis experiment. Palladium oxide particles impregnated on the surface as the co-catalyst were reduced to Pd metal during the initial hours of irradiation. The photoreduction of a noble metal co-catalyst impregnated on a catalyst surface has been reported earlier by many authors. ${ }^{19,20}$

\subsection{Characterization}

Powder X-ray diffraction (XRD) patterns of these samples were recorded using a Philips PW1820-X-ray diffractometer coupled with a PW 1729 generator, which was operated at $30 \mathrm{kV}$ and $20 \mathrm{~mA}$. A graphite crystal monochromator was used for generating monochromatic $\mathrm{CuK}_{\alpha}$ radiation. The surface area of the samples was measured using the Brunauer, Emmett and Teller (BET) method employing nitrogen as the adsorbent gas. The samples were degassed at $300{ }^{\circ} \mathrm{C}$ for $2 \mathrm{~h}$ before the measurements. Transmission electron microscopy (TEM) measurements were performed using $200 \mathrm{keV}$ electrons on a JEOL 210 UHR TEM microscope (Tokyo, Japan). Samples were dispersed in methanol and a drop of this solution was added to a carbon coated copper grid and was dried prior to loading into the TEM machine. Raman spectra were recorded using $532 \mathrm{~nm}$ lines from a diode-pumped Nd-YAG laser (power $15 \mathrm{~mW}$ ) focused onto a spot size of about $20 \mu \mathrm{m}$. The scattered light was analyzed using a home-built $0.9 \mathrm{~m}$ single monochromator coupled with an edge filter and detected using a cooled charge coupled device (CCD, Andor technology). The entrance slit was kept at $50 \mu \mathrm{m}$, which gave a resolution limited line width of $3 \mathrm{~cm}^{-1}$. DR-UV-visible spectra of all samples were recorded using a Jasco (model V-670) spectrophotometer equipped with an integrating sphere accessory. Barium sulphate was used as the reference for the reflectance spectra. Photoluminescence (PL) measurements in the visible region were carried out with an Edinburgh Instruments FLSP 920 system with a $450 \mathrm{~W}$ Xe arc lamp as the excitation source and a red sensitive Peltier element cooled Hamamatsu R2658 PMT as the detector. The emission spectra were recorded at a resolution of $1.0 \mathrm{~nm}$, by exciting the samples at $260 \mathrm{~nm}$. All the emission patterns were corrected for the detector response and were measured at $1 \mathrm{~nm}$ resolution unless otherwise stated. Excited state lifetimes of the charge carriers were measured using the same instrument and a nanosecond hydrogen flash lamp as the excitation source and employing the Time Correlated Single Photon Counting (TCSPC) technique. The frequency of the lamp was $40 \mathrm{kHz}$. The samples were excited at $282 \mathrm{~nm}$ and the lifetime was measured for $700 \mathrm{~nm}$ emission. X-ray photoelectron spectroscopy (XPS) studies were carried out on a VG Microtech electron spectrometer using $\mathrm{Mg}-\mathrm{K}_{\alpha}$ X-rays $(h \nu=1253.6 \mathrm{eV})$ as the primary source of radiation. The chamber pressure was maintained at $1 \times 10^{-9}$ torr. An appropriate correction for the charging effect was made with the help of the C 1 s signal appearing at $284.5 \mathrm{eV}$. Solid state ${ }^{29} \mathrm{Si}$ and ${ }^{27} \mathrm{Al}$ magic angle spinning nuclear magnetic resonance (MAS NMR) patterns were recorded using a Bruker Avance III $400 \mathrm{MHz}$ NMR machine with basic frequencies of 79.46 and $104.22 \mathrm{MHz}$ respectively. A single pulse experiment with a pulse duration of $3.5 \mu$ s and a relaxation delay time of 10 seconds was used for recording ${ }^{29} \mathrm{Si}$ MAS NMR patterns. The corresponding values for ${ }^{27} \mathrm{Al}$ MAS NMR experiments are $4 \mu \mathrm{s}$ and $5 \mathrm{~s}$ respectively; the samples were packed in $4 \mathrm{~mm}$ zirconia 
rotors and subjected to a spinning speed of $10 \mathrm{kHz}$ for recording the MAS NMR patterns. Chemical shift values are expressed with respect to tetramethyl silane (TMS) and $1 \mathrm{~N}$ aqueous solution of $\mathrm{Al}\left(\mathrm{NO}_{3}\right)_{3}$ for ${ }^{29} \mathrm{Si}$ and ${ }^{27} \mathrm{Al}$ nuclei respectively.

\subsection{Photocatalytic activity studies}

The photocatalytic activity was studied in a tubular quartz reactor using daylight fluorescent lamps (288 Watts) as the source of radiation. The details of the photoirradiator (S1, ESI $\dagger$ ) and the spectrum of the fluorescent lamp (Fig. S2) are given in ESI. $\dagger$ Fifty milligrams of the sample were kept in contact with aqueous solutions of $\mathrm{Na}_{2} \mathrm{~S}\left(12.5 \mathrm{~cm}^{3}\right)$ and $\mathrm{Na}_{2} \mathrm{SO}_{3}\left(12.5 \mathrm{~cm}^{3}\right)$ for conducting the photocatalysis experiment. The reactor was flushed with argon gas before irradiation. Every one hour, the gas mixture in the reactor was analyzed using a gas chromatograph (Chromatography and Instruments company, GC 2011) equipped with a molecular sieve $5 \mathrm{~A}$ column and thermal conductivity detector. The total intensity of light incident at the sample position was measured using a Gentec power meter (model Solo 2 (R2)). Details of the irradiation chamber (S1) are given in the ESI $\dagger$ and in our earlier articles. ${ }^{21,22}$

\section{Results and discussion}

\subsection{Phase identification and morphology}

The powder XRD patterns of all samples are shown in Fig. 1. It may be seen that CdS and CdPdS exist in the cubic phase (JCPDS No. 80-0019). It may be noted that in the composite too, CdS exists in the cubic phase. The XRD patterns of the composites confirm that CdS and zeolite exist as separate phases. The XRD pattern of CdPdS is very similar to that of CdS except that a very small peak around $48.1^{\circ}$ is seen. This peak can be due to the presence of a very small amount of hexagonal CdS. No additional peak corresponding to PdS is seen indicating that $\mathrm{Pd}^{2+}$ has substituted for $\mathrm{Cd}^{2+}$ in the lattice of CdS. The BET surface area values obtained for NaY zeolite, CdS, CdPdS, 10CdS-Z, 20CdS-Z, 30CdS-Z, 50CdS-Z and 20CdPdS-Z are 670, 45, 48, 168, 154, 103, 82 and $143 \mathrm{~m}^{2} \mathrm{~g}^{-1}$ respectively.

To further investigate the different phases present in these samples, Raman spectra were recorded and are shown in Fig. 2. Pure CdS shows Raman bands around 306 and $610 \mathrm{~cm}^{-1}$, which can be assigned to the 1 LO (longitudinal optical) and 2 LO phonon modes of CdS respectively. ${ }^{23}$ The Raman spectrum of zeolite shows a broad band at $503 \mathrm{~cm}^{-1}$, which is characteristic of Y-zeolite. ${ }^{24}$ The Raman spectra of CdPdS and 20CdPdS-Z are identical to that of CdS and no band corresponding to any other phase like PdS or PdO is seen, which is in conformity with our XRD results. Besides, the Raman bands due to zeolite are not seen in the composite as the Raman spectrum of zeolite is, in general, dominated by a high background fluorescence due to defects. $^{25}$ The strong background fluorescence makes the Raman bands weak or completely undetectable. A similar effect of the non-observance of zeolite bands in the Raman spectra of the CdS-zeolite composite has been reported previously. ${ }^{26,27}$

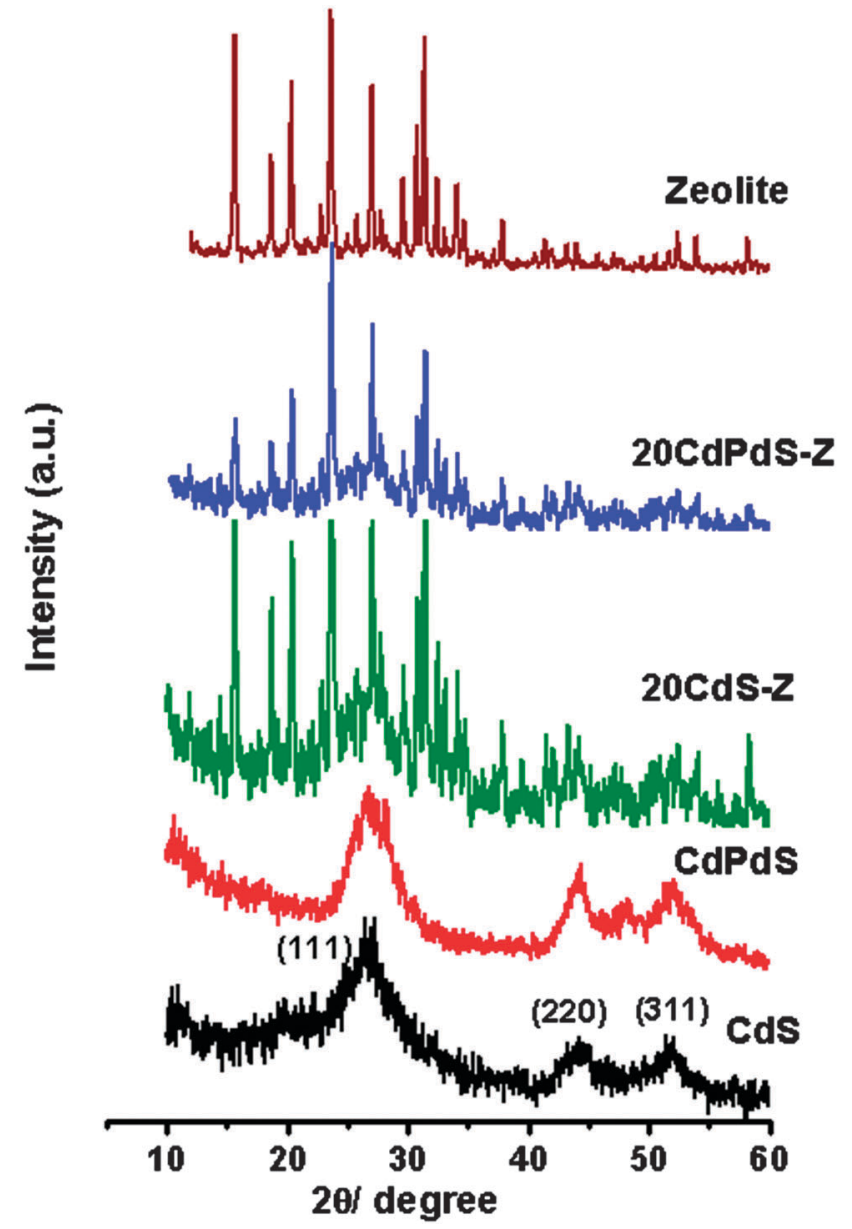

Fig. 1 Powder XRD patterns of CdS, CdPdS, 20CdS-Z, 20CdPdS-Z and NaY-zeolite.

TEM and HRTEM images along with the SAED pattern of CdPdS are shown in Fig. 3A. It is seen from the figure that CdPdS exists as nanosized particles with a size of $20-30 \mathrm{~nm}$. The distance between the fringes obtained from the HRTEM image of the CdPdS sample matched well with the (111) plane of cubic CdS. The SAED pattern could also be indexed as cubic CdS. The TEM image and SAED pattern of 20CdPdS-Z are shown in Fig. 3B. The image consists of irregular shaped, large sized aggregated particles. More TEM images recorded from different regions are shown in Fig. S3 of the ESI. $\dagger$ SAED patterns recorded from different regions of the aggregated particles, showed a diffraction pattern characteristic of the crystalline Y-zeolite phase. No SAED patterns corresponding to CdS phase could be obtained. However, the presence of CdS in this composite is confirmed from the XRD pattern and the Raman spectrum. Thus, the TEM studies suggest that CdS exists as a highly dispersed phase on zeolite.

\subsection{Optical properties}

UV-visible DRS of CdS, zeolite and the composites are shown in Fig. 4A. A blue shift of the absorption edge is seen for the composite samples compared to CdS. The spectrum of zeolite does not show any absorption as it is a wide bandgap material. Pd doping in CdS results in a significant increase in visible light 


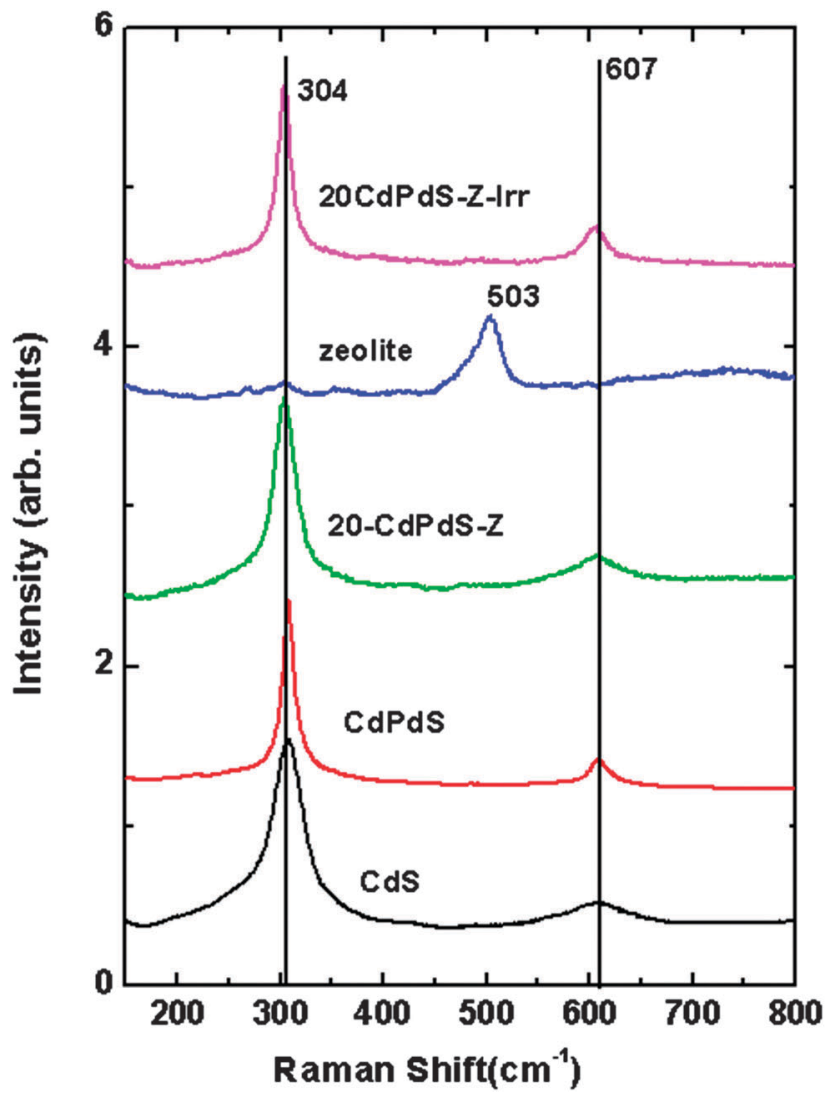

Fig. 2 Raman spectra of CdS, CdPdS, NaY-zeolite, 20CdPdS-Z and 20CdPdS-Z-Irr (after the photocatalysis experiment).

absorption as seen from the spectrum of CdPdS and the 20CdPdS-Z composite. Fig. 4B shows the modified KubelkaMunk function $\left[\left(F\left(R_{\infty}\right) h \nu\right)^{2}\right]$ plotted against $h \nu$. The bandgaps of $\mathrm{CdS}$ and the composites obtained from these plots are 2.40, 1.16, 2.25, 2.49 and 2.53 for CdS, CdPdS, 20CdPdS-Z, 30CdS-Z and $20 \mathrm{CdS}-Z$ respectively. Thus, a clear decrease in the bandgap of CdS is observed as a result of Pd doping in CdS.

The PL spectra of CdS, zeolite, CdPdS, 20CdS- $Z$ and 20CdPdS-Z are shown in Fig. 5A. The emission spectrum of CdS shows a broad peak around $590 \mathrm{~nm}$. The wavelength corresponding to the emission peaks of CdS suggests that the peak is originating from the defect emission. ${ }^{28}$ Pure zeolite shows a broad peak centered at $440 \mathrm{~nm}$ and can be assigned to the defect emission. The spectra of the composites are similar and show the defect emission peaks corresponding to both CdS and zeolite. The PL spectrum of CdPdS did not show any peak as it is dark in color and hence no emission occurs from it.

Fluorescence lifetime studies were carried out for the CdSzeolite composite samples and the time resolved fluorescence decay plots are shown in Fig. 5B. In powder samples, the intensity of the emission peak depends on the properties of the powder and hence it is incorrect to draw any conclusion regarding the luminescence efficiency or lifetime based on a comparison of the intensities of the emission peaks. Fluorescence lifetime is the more appropriate parameter that needs to be considered for this purpose. The fluorescence emission

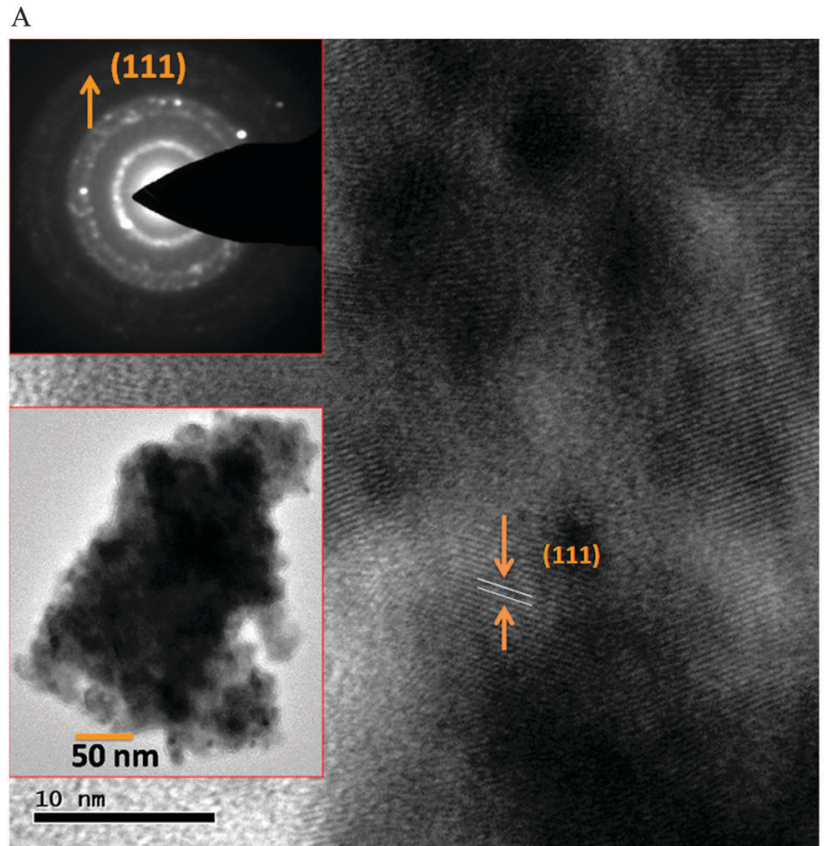

B

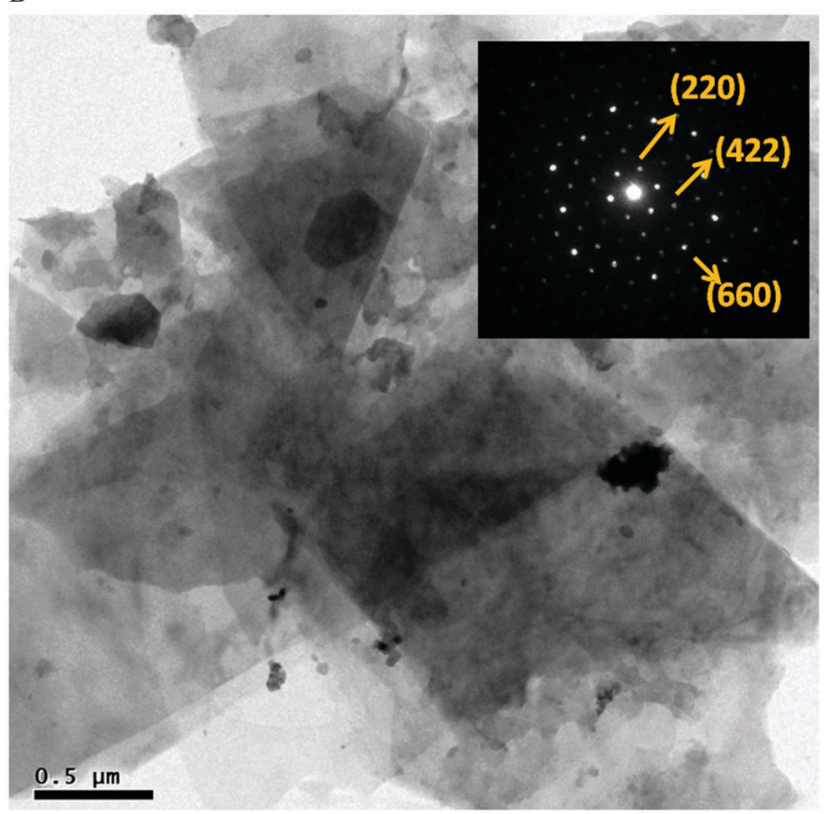

Fig. 3 (A) TEM, HRTEM and SAED patterns of CdPdS and (B) TEM and SAED patterns of $20 \mathrm{CdPdS}-Z$.

decay at $590 \mathrm{~nm}$ was studied. It can be seen from the figure that the recombination is slower in the composite samples than in pristine CdS. The fluorescence lifetimes obtained for CdS, 20CdS-Z and 20CdPdS-Z are 193, 603 and 620 ps respectively. The increased lifetime of the charge carriers in the composite samples indicates that zeolite is playing a role in increasing the lifetime of the photogenerated charges.

\subsection{XPS studies}

X-ray photoelectron spectra of 20CdPdS-Z before and after photocatalysis experiments were recorded to determine the 

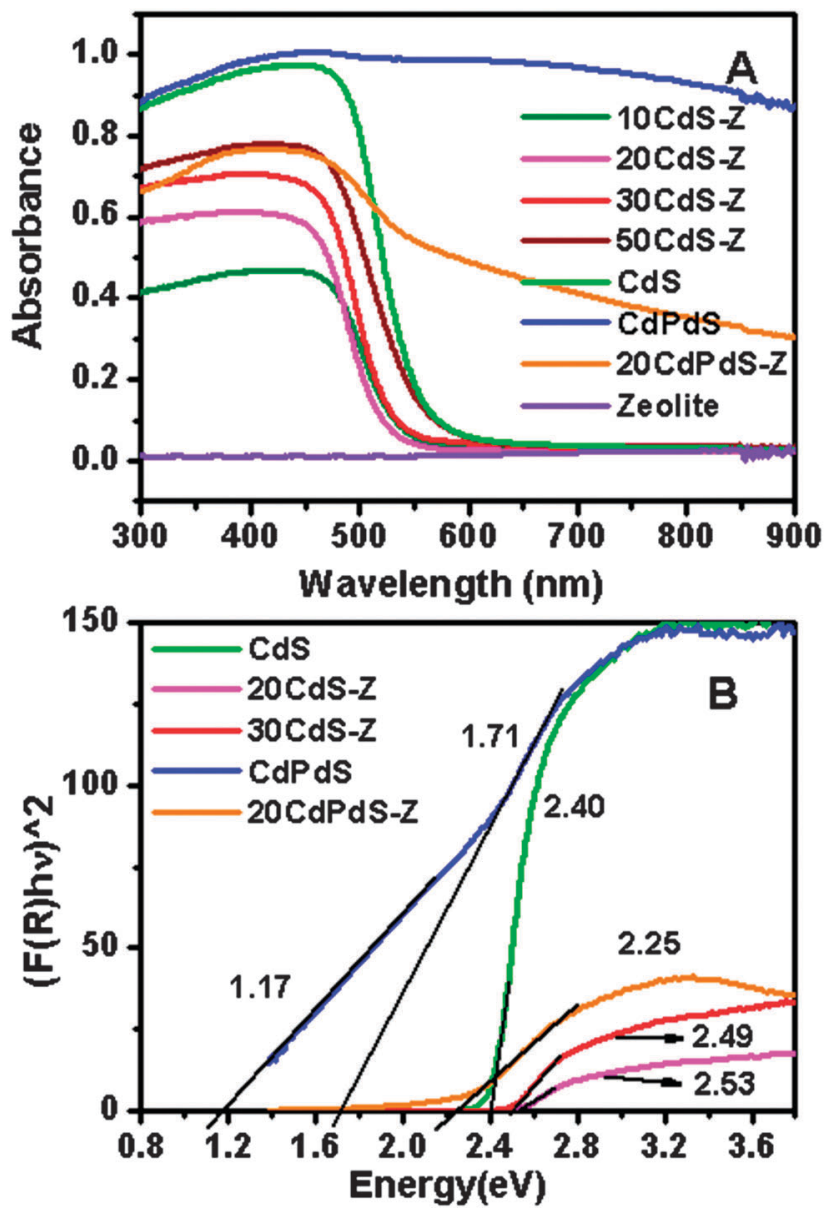

Fig. 4 (A) UV-Visible DRS of CdS, zeolite and composites (B) Modified $\mathrm{K}-\mathrm{M}$ function plotted against $h \nu$ for $\mathrm{CdS}$ and composites.

oxidation state of the different species present on the surface and to see whether any change in the oxidation state occurred after catalysis. The Pd 3d spectra of these samples are shown in Fig. 6. It can be seen that the spectra of both 20CdPdS-Z composites before and after catalysis showed two peaks at 336.1 and $341.2 \mathrm{eV}$. These peaks can be assigned to the $3 \mathrm{~d}_{5 / 2}$ and $3 \mathrm{~d}_{3 / 2}$ spin orbital components of the $\mathrm{Pd}^{2+}$ species. ${ }^{29} \mathrm{It}$ is seen that peak position is more or less the same in both samples. Even after irradiation, there is no significant change in the peak positions, indicating that palladium remains as the oxidized species in the doped samples. The S2p spectra of 20CdPdS-Z are shown in Fig. 7. The spectra of original and irradiated samples showed a single peak with a binding energy of $161.8 \mathrm{eV}$ and the peak position is unchanged for the irradiated sample. This corresponds to the $\mathrm{S}^{2-}$ of cadmium sulfide. ${ }^{30}$ The Cd $3 \mathrm{~d}$ spectra of all samples are shown in Fig. 7. Two peaks seen at 404.1 and $410.9 \mathrm{eV}$ arise from the $3 \mathrm{~d}_{5 / 2}$ and $3 \mathrm{~d}_{3 / 2}$ components of $\mathrm{Cd}$ in CdS. ${ }^{31}$ The surface composition of 20CdPdS-Z as obtained from the XPS analysis before and after photocatalysis work is shown in Table 1 . It can be seen that there is no significant change in the composition of CdPdS after photocatalysis work indicating that the catalyst is quite stable under the present experimental conditions.
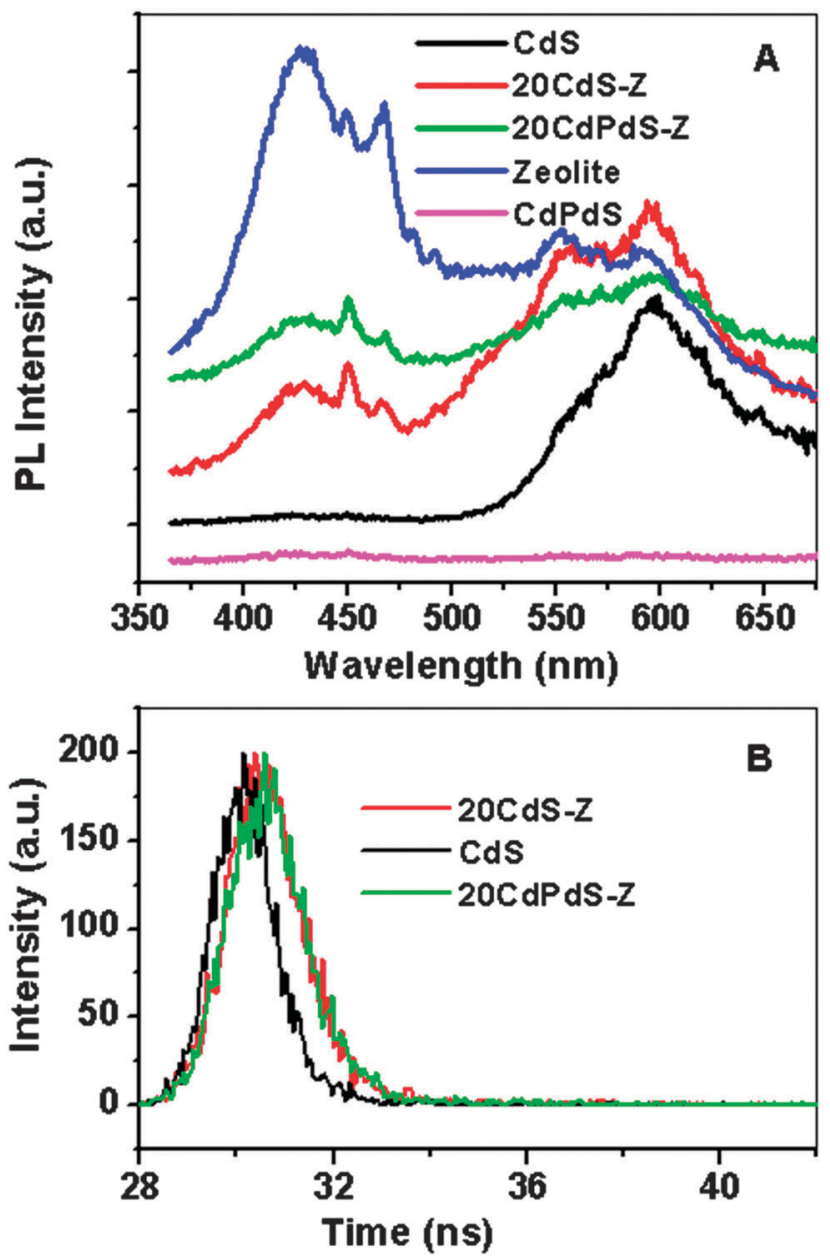

Fig. 5 (A) Photoluminescence spectra of CdS, CdPdS, zeolite and composites; (B) fluorescence emission decay curves (emission at $590 \mathrm{~nm}$ ) for CdS, 20CdS-Z and 20CdPdS-Z.

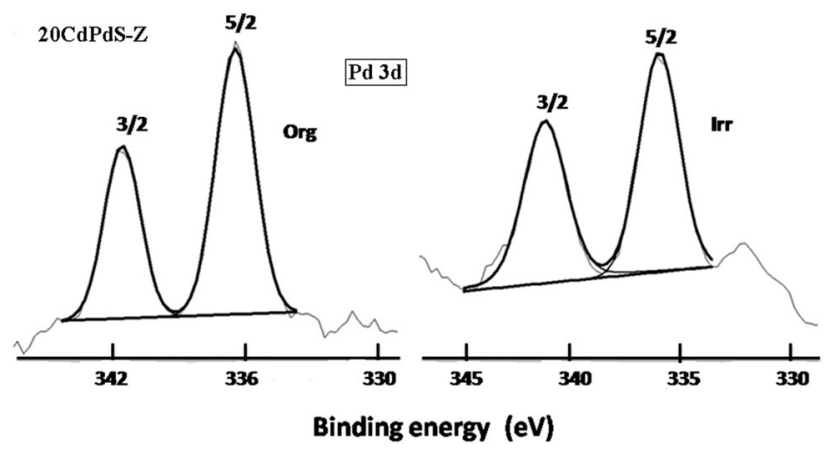

Fig. $6 \mathrm{Pd} 3 \mathrm{~d}$ X-ray photoelectron spectra of $20 \mathrm{CdPdS}-Z$ of the original and irradiated samples.

\subsection{Solid state MAS NMR studies}

The ${ }^{27} \mathrm{Al}$ and ${ }^{29} \mathrm{Si}$ NMR spectra of zeolite, $20 \mathrm{CdS}-\mathrm{Z}$ and 20CdPdS-Z were recorded to study the nature of the interaction between CdS and CdPdS with zeolite. Fig. 8 shows the ${ }^{27} \mathrm{Al}$ NMR spectra of NaY zeolite, 20CdS-Z and 20CdPdS-Z. A peak around $62 \mathrm{ppm}$ is seen for all samples indicating that $\mathrm{Al}$ is in the 


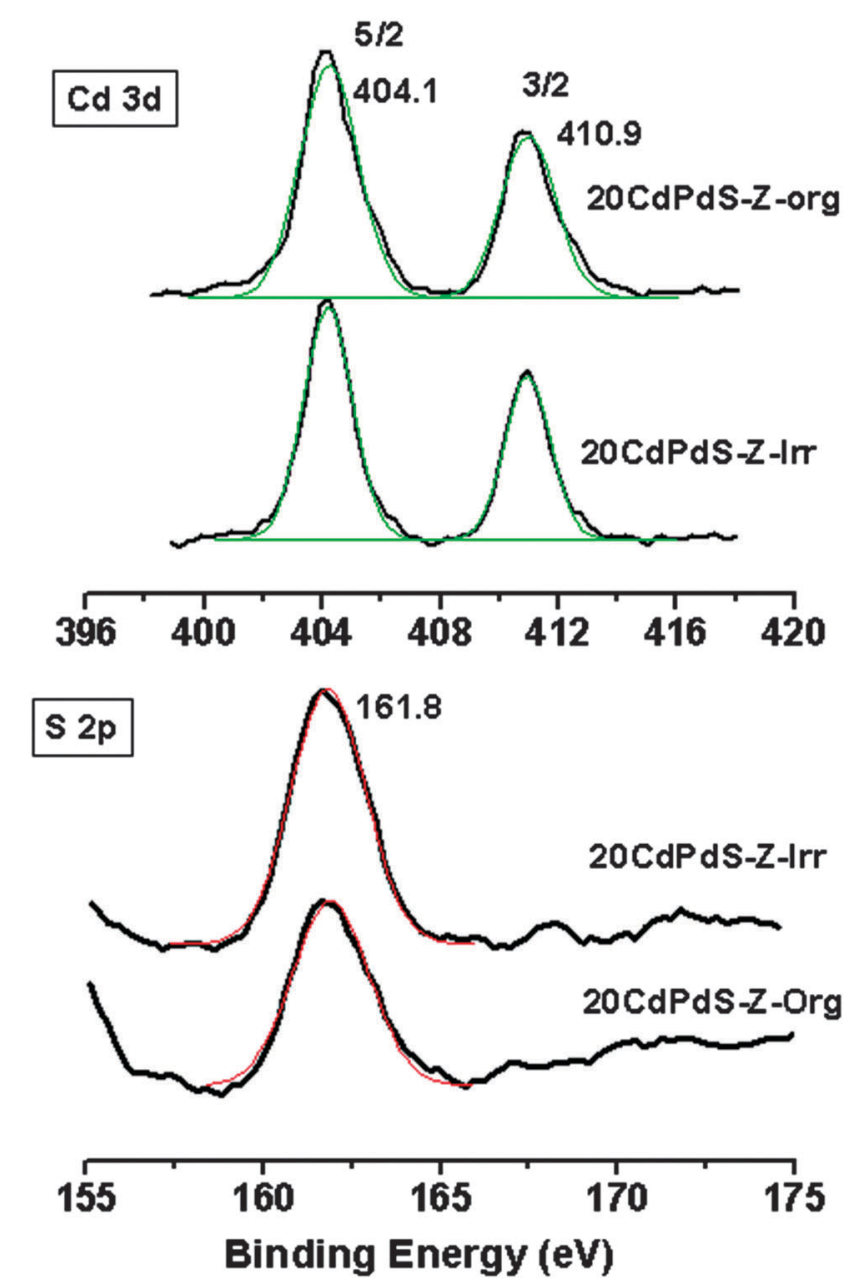

Fig. $7 \mathrm{Cd} \mathrm{3d}$ and S $2 p$ X-ray photoelectron spectra of $20 \mathrm{CdPdS}-Z$ of the original and irradiated samples.

Table 1 Surface composition of 20CdPdS-Z samples before and after photocatalysis obtained from XPS analysis

\begin{tabular}{lcc}
\hline Element & 20CdPdS-Z-Org (atom\%) & 20CdPdS-Z-Irr (atom\%) \\
\hline Cd & 14.5 & 16.4 \\
Pd & 3.6 & 3.1 \\
S & 12.9 & 13.3 \\
C & 12.2 & 7.7 \\
O & 37.6 & 40.7 \\
Si & 14.3 & 13.4 \\
Al & 4.9 & 5.4
\end{tabular}

4-coordination state. ${ }^{32,33} \mathrm{~A}$ closer look at the peaks indicates that there is a shift of the peak towards lower chemical shift values for the composite samples. Though, the shift of the peak position of 20CdPdS-Z appears to be small compared to zeolite, it is significant as ${ }^{27} \mathrm{Al}$ is a quadrupolar nucleus and its sensitivity to the electronic environment is relatively less compared to the spin $1 / 2$ nucleus. This shift can be explained by considering the fact that with $\mathrm{Cd}^{2+}$ incorporation in the system, the $\mathrm{Na}^{+}$attached with $\mathrm{AlO}_{4}{ }^{-}$units gets replaced with $\mathrm{Cd}^{2+}$ ions. As the $\mathrm{Z} / \mathrm{r}$ value of $\mathrm{Cd}^{2+}(2.6)$ is much larger than $\mathrm{Na}^{+}$ (1.01, almost double), the $\mathrm{AlO}_{4}{ }^{-}-\mathrm{Cd}^{2+}$ interaction will be more

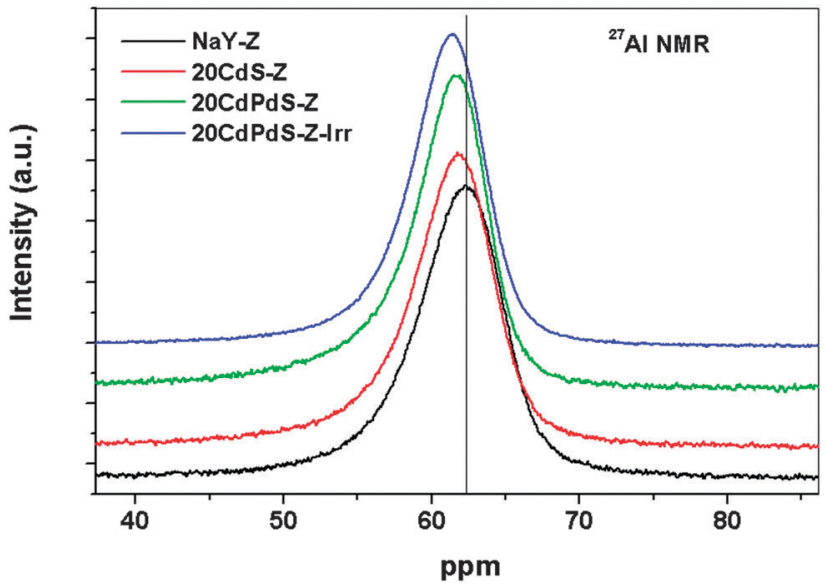

Fig. $8 \quad .{ }^{27} \mathrm{Al}$ MAS NMR spectra of NaY-zeolite, 20CdS-Z, 20CdPdS-Z and $20 \mathrm{CdPdS}-\mathrm{Z}-$ Irr (after the photocatalysis experiment).

covalent compared to the $\mathrm{AlO}_{4}{ }^{-}-\mathrm{Na}^{+}$interaction. The increased covalent character of $\mathrm{AlO}_{4}{ }^{-}-\mathrm{Cd}^{2+}$ linkages results in increased electron density in $\mathrm{AlO}_{4}{ }^{-}$structural units and eventually increases the net electron density around Al. This leads to decreased ${ }^{27} \mathrm{Al}$ NMR chemical shift values for $\mathrm{AlO}_{4}{ }^{-}-\mathrm{Cd}^{2+}$ linkages compared to $\mathrm{AlO}_{4}^{-}-\mathrm{Na}^{+}$linkages and an associated upfield shift in the ${ }^{27} \mathrm{Al}$ MAS NMR peak. The ${ }^{29} \mathrm{Si}$ MAS NMR spectra of all samples are shown in Fig. 9. The ${ }^{29} \mathrm{Si}$ MAS NMR pattern of the Zeolite Y sample is characterized mainly by four peaks around $-105,-99,-93$ and $-88 \mathrm{ppm}$ which are characteristic of silicon structural units having different number of $\mathrm{Al}^{3+}$ ions as next nearest neighbors. Based on the previous ${ }^{29} \mathrm{Si}$ MAS NMR studies of Zeolite Y samples, the peaks around $-105,-99$, -93 and $-88 \mathrm{ppm}$ have been attributed to silicon structural units with $1 \mathrm{Al}, 2 \mathrm{Al}, 3 \mathrm{Al}$ and $4 \mathrm{Al}$ respectively as the next nearest neighbors. ${ }^{34}$ The chemical shift values of peaks corresponding to all samples are almost the same indicating that the local environment around $\mathrm{Si}$ in the framework is unaffected by the dispersion of CdS (or CdPdS) on its surface and there is no direct interaction between silicon structural units and $\mathrm{Cd}^{2+}$ ions. Thus, ${ }^{29} \mathrm{Si}$ MAS NMR results substantiate the ${ }^{27} \mathrm{Al}$ MAS NMR results that $\mathrm{Cd}$ directly and selectively interacts with the $\mathrm{Al}$ of the zeolite framework. It may be noted that the ${ }^{27} \mathrm{Al}$ and ${ }^{29} \mathrm{Si}$ spectra of the catalyst after irradiation is very similar to the original one suggesting that the catalyst is stable and no change occurs in the catalyst during irradiation.

\subsection{Photocatalytic activity and the possible electron transfer process in the composite}

Fig. 10A shows the variation of the photocatalytic activity of the CdS-zeolite composite as the concentration of CdS on zeolite is increased. It is seen that the photocatalytic activity initially increases with CdS loading and reaches a maximum when the concentration is $20 \%$. Beyond that, the activity decreases indicating that the dispersion of CdS is the highest at the $20 \%$ concentration of CdS. Further increase in the concentration of CdS decreases the effective surface area and results in a decrease in the photocatalytic activity. As expected, zeolite does not show 


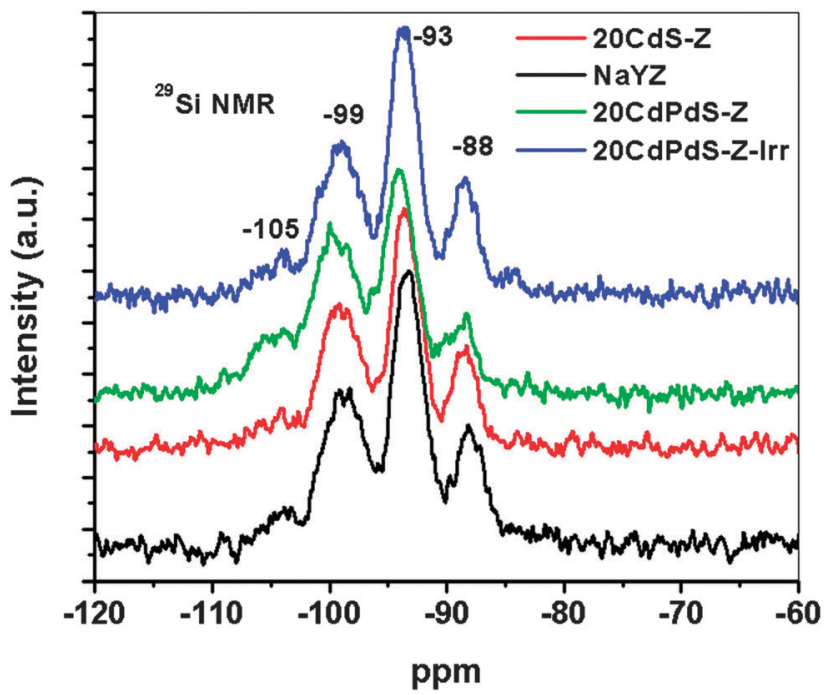

Fig. $9{ }^{29}$ Si MAS NMR spectra of NaY-zeolite, 20CdS-Z, 20CdPdS-Z and 20CdPdS-Z-Irr.

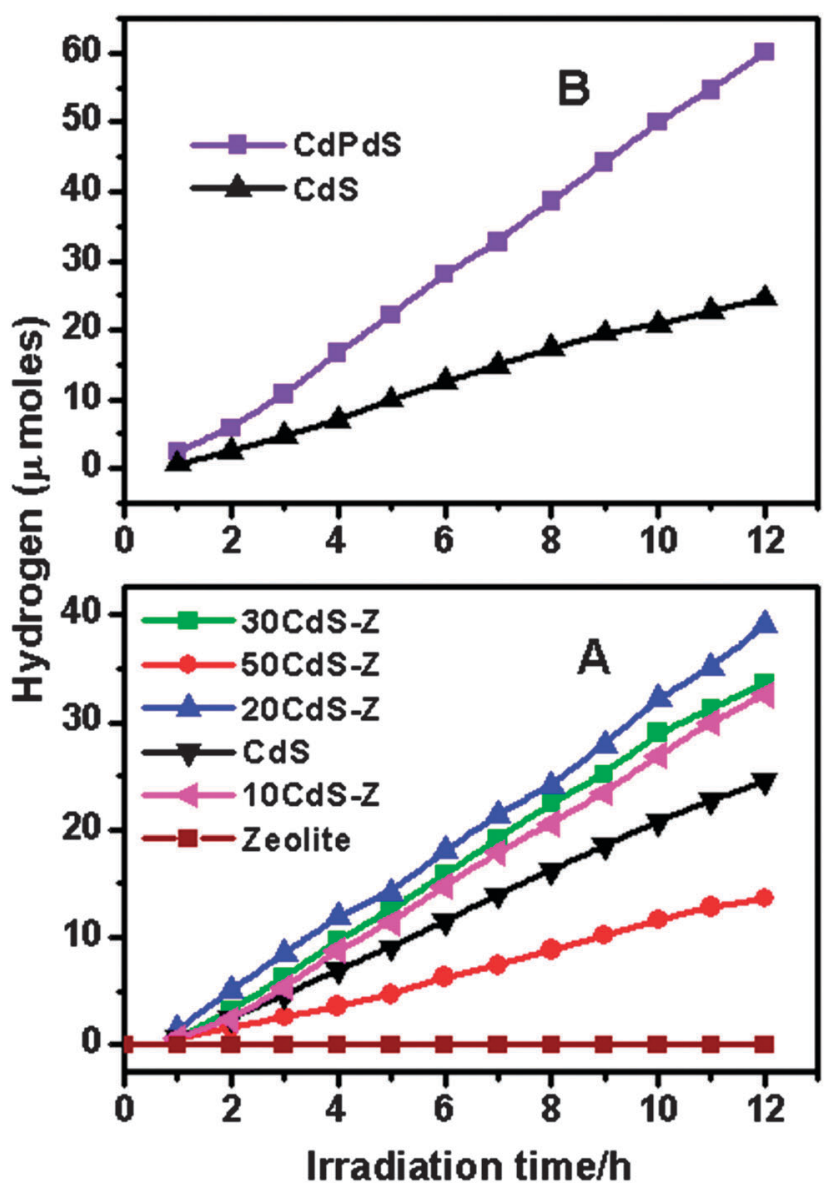

Fig. 10 Photocatalytic activity for hydrogen generation as a function of irradiation time for: (A) CdS, zeolite and CdS-zeolite composites with different compositions (B) CdS and CdPdS. Reaction conditions: $50 \mathrm{mg}$ catalyst suspended in $25 \mathrm{~cm}^{3}$ of aqueous solution containing $\mathrm{Na}_{2} \mathrm{SO}_{3}$ $(0.8 \mathrm{M})$ and $\mathrm{Na}_{2} \mathrm{~S}(0.6 \mathrm{M})$ in $1: 1$ ratio by volume; light source: ordinary day light fluorescent lamp (total $36 \times 8=288$ watts). Amount of hydrogen given in the figure is normalized to $50 \mathrm{mg}$ of $\mathrm{CdS}$ for composites as the amount of pure CdS taken for the experiment is $50 \mathrm{mg}$. any activity due to its wide bandgap. The effect of Pd doping in CdS on the photocatalytic activity is shown in Fig. 10B. A considerable increase in the photocatalytic activity for CdS is seen after doping with Pd. This increase in the photocatalytic activity of CdPdS can be attributed to the improved visible light absorption as seen from the UV-visible DRS of this sample. The effects of loading $\mathrm{Pd}$ as the co-catalyst on the photocatalytic activity of CdPdS and 20CdPdS-Z are shown in Fig. 11. It can be seen that the presence of the Pd co-catalyst enhances the photocatalytic activity of both CdPdS and 20CdPdS-Z and a hydrogen generation rate of $113 \mu \mathrm{mol} \mathrm{h}{ }^{-1}$ is obtained for the Pd-20CdPdS-Z catalyst. These results clearly reveal that the presence of palladium as both dopant and co-catalyst plays a significant role in increasing the photocatalytic activity of CdS dispersed on zeolite.

The stability of the catalyst was tested by repeating the photocatalysis experiment using the same catalyst after flushing the system with argon gas. The time course of hydrogen evolution for repeated cycles is shown in Fig. 12. It is seen that there is no significant decrease in the photocatalytic activity up to $60 \mathrm{~h}$ in the experiment indicating that the catalyst is stable under the present experimental conditions. The XRD patterns of the samples recorded before and after the photocatalysis work (Fig. S4 of the ESI $\dagger$ ) indicate that no phase change occurs in the sample during photocatalytic reaction. Besides, the Raman spectrum of the irradiated 20CdPdS-Z sample (Fig. 2) is similar

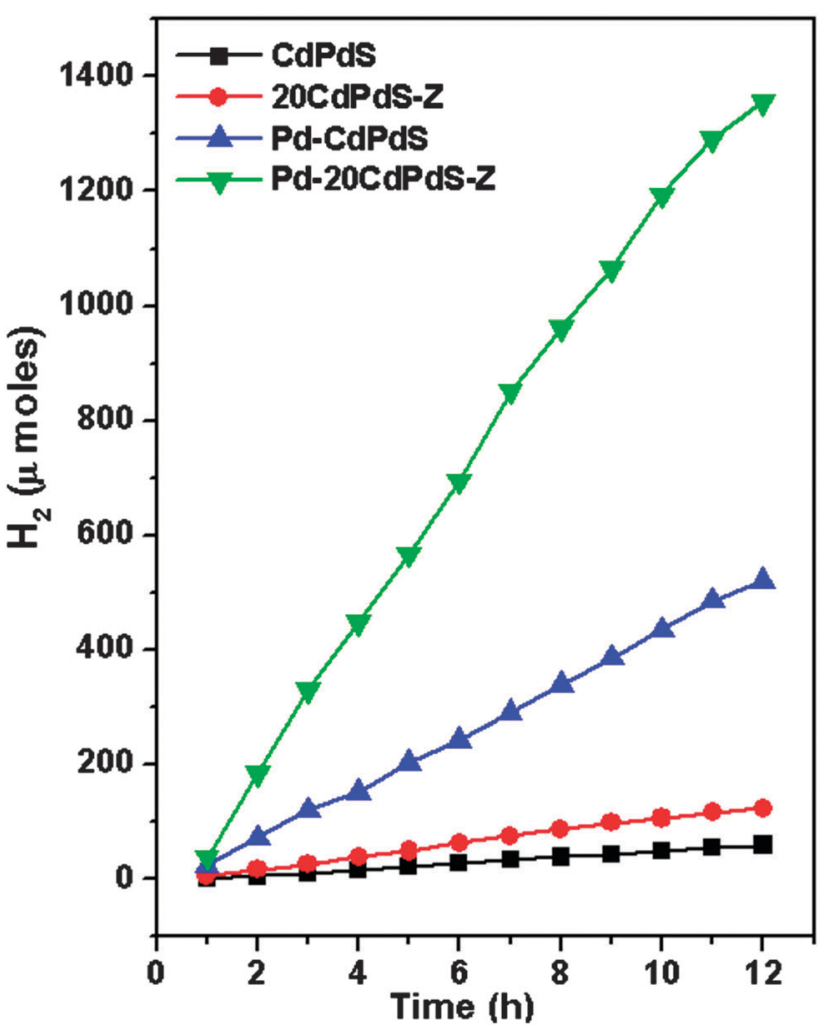

Fig. 11 Photocatalytic activity for hydrogen generation as a function of irradiation time for CdPdS, 20CdPdS, Pd impregnated 20CdPdS and $20 \mathrm{CdPdS}-\mathrm{Z}$. Amount of hydrogen given in the figure is normalized to $50 \mathrm{mg}$ of $\mathrm{CdS}$ for composites. 


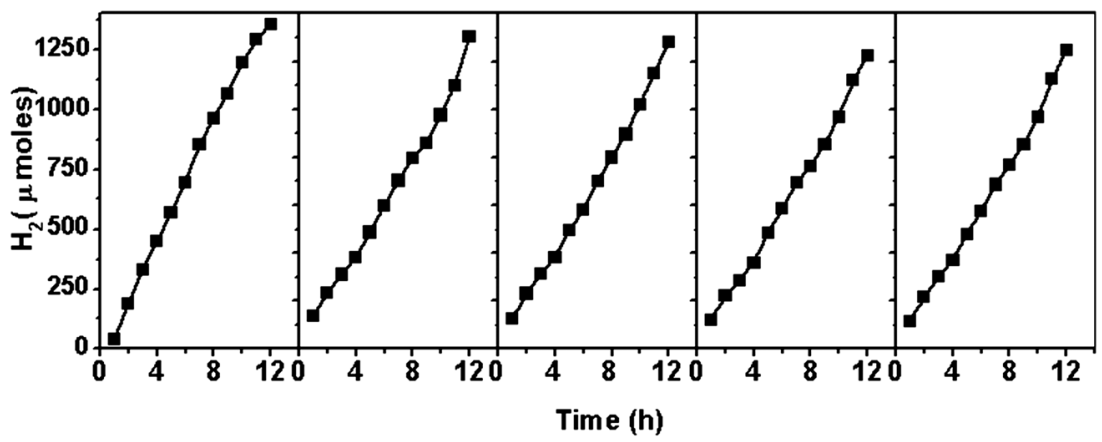

Fig. 12 Photocatalytic activity of Pd impregnated 20CdPdS-Z for repeated cycles (reaction conditions same as that of the previous experiment).

to that of the fresh catalyst, which further confirms that no noticeable change occurs in the catalyst due to irradiation. It may be recalled that the surface oxidation state of different elements and the composition of the 20CdPdS-Z are also found to be almost the same for the original and irradiated samples from the XPS spectra. Thus, both the surface and bulk of 20CdPdS-Z remain unchanged after the photocatalysis experiment.

Photocatalytic activity studies clearly reveal that zeolite support plays a prominent role in increasing the photocatalytic activity of CdS. The enhanced activity is arising due to two reasons. Dispersion of CdS on zeolite increases the surface area of CdS preventing aggregation, which increases the photocatalytic activity. Besides, the polar environment of zeolite plays a role in improving the charge separation. It acts as a media for charge transfer owing to its electron acceptor-donor properties. Hence, the photogenerated electrons from CdS (or CdPdS) can be effectively transferred to the zeolite framework resulting in an increased lifetime of the charge carriers. ${ }^{35-37}$ This view is supported by the fluorescence lifetime studies of CdS-zeolite composites. For the charge transfer from CdS to zeolite to be effective, an intimate contact between CdS and zeolite is

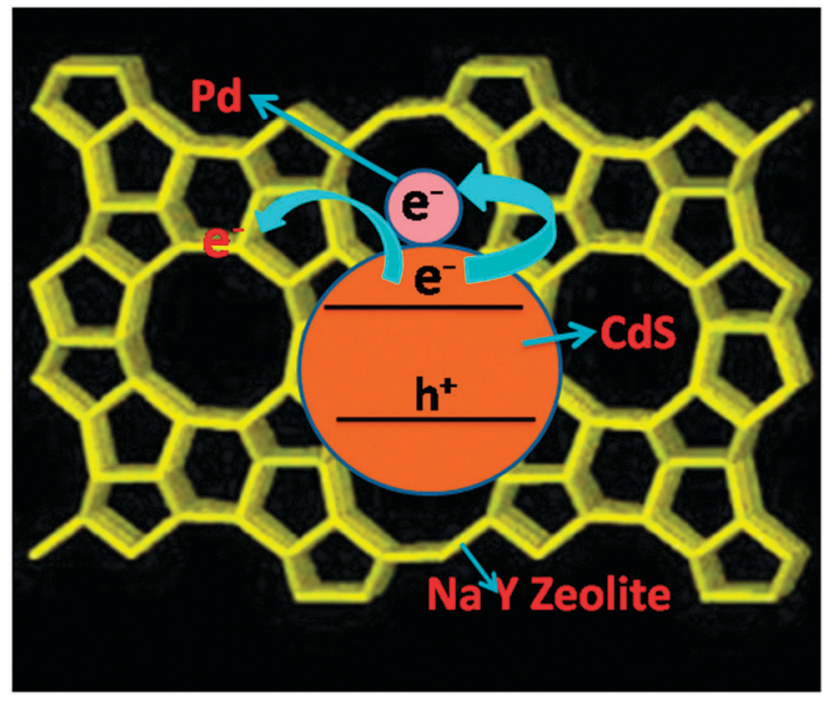

Fig. 13 Schematic illustration of the photogenerated charge separation process in the $\mathrm{Pd}-\mathrm{CdS}$-zeolite composite. needed. The NMR spectra of the composite samples clearly show the interaction between CdS and zeolite at the interface, which can favor the charge transfer from CdS to zeolite.

Further, the present study shows that palladium can play multiple roles in enhancing the photocatalytic activity of CdS. Palladium as $\mathrm{Pd}^{2+}$ in CdPdS enhances the optical absorption property by altering the band structure of CdS and improves the photocatalytic activity. Palladium as the co-catalyst $\left(\mathrm{Pd}^{0}\right)$ improves the activity further by increasing the lifetime of charge carriers. The presence of Pd metal in the palladium impregnated sample could not be seen from the XPS of this sample as its concentration was very low ( $0.5 \%$ by weight). But, its presence is discerned from the photocatalytic activity studies, which clearly show an enhanced activity after Pd impregnation. This is in conformity with the studies reported earlier by us as well as by many other authors. ${ }^{5,11,20-22,38,39}$ When Pd is used as a co-catalyst, interfacial transfer of electrons occurs from the photocatalyst to Pd. Through this process, the charges are separated efficiently and recombination is reduced. The increased availability of electrons at the metal sites increases the photocatalytic efficiency. A schematic illustration of the charge separation process occurring in the Pd-CdS-zeolite composite is shown in Fig. 13.

\section{Conclusions}

Palladium doped CdS dispersed on zeolite with Pd as the co-catalyst (Pd-20CdPdS-Z) exhibits enhanced photocatalytic activity for hydrogen generation from water compared to pure CdS, palladium doped CdS and CdS dispersed on zeolite. CdS and CdPdS interact selectively with $\mathrm{Al}$ of the zeolite framework. In this system, Pd plays the dual role of improving the visible light absorption and increasing the lifetime of the photogenerated electrons. Palladium doping in CdS improves the optical absorption properties of CdS whereas zeolite and the Pd metal co-catalyst increase the lifetime of the charge carriers by enhancing the interfacial transfer of electrons from CdS to Pd metal and zeolite. This observation is supported by the increased fluorescence lifetime for the charge carriers in the composite system. The catalyst is found to be stable and the photocatalytic activity is unchanged for repeated cycles of the experiment. 


\section{References}

1 N. Bao, L. Shen, T. Takata, K. Domen, A. Gupta, K. Yanagisawa and C. A. Grimes, J. Phys. Chem. C, 2007, 111, 17527-17534.

2 H. Park, Y. K. Kim and W. Choi, J. Phys. Chem. C, 2011, 115, 6141-6148.

3 G. C. De, A. M. Roy and S. S. Bhattacharya, Int. J. Hydrogen Energy, 1996, 21, 19-23.

4 K. He, M. Li and L. Guo, Int. J. Hydrogen Energy, 2012, 37, 755-759.

5 R. Sasikala, A. R. Shirole, V. Sudarsan, K. G. Girija, R. Rao, C. Sudakar and S. R. Bharadwaj, J. Mater. Chem., 2011, 21, 16566-16573.

6 M. Sathish, B. Viswanathan and R. P. Viswanath, Int. J. Hydrogen Energy, 2006, 31, 891-898.

7 X. Wang, G. Liu, Z. G. Chen, F. Li, L. Wang, G. Q. Lu and H. M. Cheng, Chem. Commun., 2009, 3452-3454.

8 W. Yang, Y. Liu, Y. Hu, M. Zhou and H. Qian, J. Mater. Chem., 2012, 22, 13895.

9 S. V. Kahane, R. Sasikala, B. Vishwanadh, V. Sudarsan and S. Mahamuni, Int. J. Hydrogen Energy, 2013, 38, 15012-15018.

10 X. An, X. Yu, J. C. Yu and G. Zhang, J. Mater. Chem. A, 2013, 1, 5158-5164.

11 F. N. Sayed, R. Sasikala, O. D. Jayakumar, R. Rao, C. A. Betty, A. Chokkalingam, R. M. Kadam, Jagannath, S. R. Bharadwaj, A. Vinu and A. K. Tyagi, RSC Adv., 2014, 4, 13469-13476.

12 M. Q. Yang, N. Zhang, M. Pagliaro and Y. J. Xu, Chem. Soc. Rev., 2014, 43, 8240-8254.

13 N. Zhang, Y. Zhang and Y. J. Xu, Nanoscale, 2012, 4, 5792-5813.

14 N. Zhang, M. Q. Yang, Z. R. Tang and Y. J. Xu, ACS Nano, 2014, 8, 623-633.

15 C. Han, M. Q. Yang, N. Zhang and Y. J. Xu, J. Mater. Chem. A, 2014, 2, 19156-19166.

16 L. Jia, D. H. Wang, Y. X. Huang, A. W. Xu and H. Q. Yu, J. Phys. Chem. C, 2011, 115, 11466-11473.

17 D. H. Wang, L. Wang and A. W. Xu, Nanoscale, 2012, 4, 2046-2053.

18 C. Han, M. Q. Yang, B. Weng and Y. J. Xu, Phys. Chem. Chem. Phys., 2014, 16, 16891-16903.

19 H. Kato, H. Kobayashi and A. Kudo, J. Phys. Chem. B, 2002, 106, 12441-12447.
20 R. Sasikala, V. Sudarsan, C. Sudakar, R. Naik, L. Panicker and S. R. Bharadwaj, Int. J. Hydrogen Energy, 2009, 34, 6105-6113.

21 R. Sasikala, A. R. Shirole, V. Sudarsan, Jagannath, C. Sudakar, R. Naik, R. Rao and S. R. Bharadwaj, Appl. Catal., A, 2010, 377, 47-54.

22 R. Sasikala, A. R. Shirole and S. R. Bharadwaj, J. Colloid Interface Sci., 2013, 409, 135-140.

23 P. Chawla, S. P. Lochab and N. Singh, J. Alloys Compd., 2010, 492, 662-666.

24 J. Twu, P. K. Dutta and C. T. Kresge, Zeolites, 1991, 11, 672.

25 H. Kim, K. M. Kosuda, R. P Van Duyne and P. C. Stair, Chem. Soc. Rev., 2010, 39, 4820-4844.

26 H. V Garcia, M. H. Velez, O. S. Garrido, J. M. M. Duart and J. Jimenez, IEE J. Solid-State Electron Devices, 1999, 43, 1171-1175.

27 M. M. Garcia, H. Villavicencio, M. H. Velez, O. Sanchez and J. M. M. Duart, Mater. Sci. Eng., C, 2001, 15, 101-104.

28 B. Yang, J. E. Schneeloch, Z. Pan, M. Furis and M. Achermann, Phys. Rev. B: Condens. Matter Mater. Phys., 2010, 81, 073401.

29 R. V. Gulyaev, E. M. Slavinskaya, S. A. Novopashin, D. V. Smovzh, A. V. Zaikovskii, D. Yu. Osadchii, O. A. Bulavchenko, S. V. Korenev and A. I. Boronin, Appl. Catal., B, 2014, 147, 132-143.

30 F. Xiangqian, L. Jinyun, W. Yuteng, Z. Xiaoman, M. Fanli and L. Jinhuai, J. Mater. Chem., 2012, 22, 17782-17791.

31 S. A. Vanalakar, R. C. Pawar, M. P. Suryawanshi, S. S. Mali, D. S. Dalavi, A. V. Moholkar, K. U. Sim, Y. B. Kown, J. H. Kim and P. S. Patil, Mater. Lett., 2011, 65, 548-551.

32 L. A. O'Dell, S. L. P. Savin, A. V. Chadwick and M. E. Smith, Solid State Nucl. Magn. Reson., 2007, 31, 169-173.

33 R. Sasikala, V. Sudarsan and S. K. Kulshreshtha, J. Solid State Chem., 2002, 169, 113-117.

34 J. Klinowski, Annu. Rev. Mater. Sci., 1988, 18, 189-218.

35 Y. Kim and M. Yoon, J. Mol. Catal. A: Chem., 2001, 168, 257-263.

36 M. Khatamian, M. S. Oskoui and M. Haghighi, New J. Chem., 2014, 38, 1684-1693.

37 R. Sasikala, A. R. Shirole, V. Sudarsan, V. S. Kamble, C. Sudakarb, R. Naik, R. Rao and S. R. Bharadwaj, Appl. Catal., A, 2010, 390, 245-252.

38 W. Sun, S. Zhang, Z. Liu, C. Wang and Z. Mao, Int. J. Hydrogen Energy, 2008, 33, 1112.

39 P. V. Kamat, J. Phys. Chem. C, 2007, 111, 2834-2860. 